\title{
CPTAC Codelists Terminology
}

National Cancer Institute

\section{Source}

National Cancer Institute. CPTAC Codelists Terminology. NCI Thesaurus. Code C158520.

Terminology used to support the various lists of data useful for tracking trends by CPTAC. 